\title{
Center Concepts on Distance $k$-Dominating Sets
}

\author{
Dr. A. Anto Kinsley \\ Department of Mathematics \\ St.Xavier's (Autonomous) College \\ Palayamkottai-627002, India
}

\author{
V. Annie Vetha Joeshi \\ Department of Mathematics \\ St.Xavier's (Autonomous) College \\ Palayamkottai-627002, India
}

\begin{abstract}
A set $\boldsymbol{D} \subseteq \boldsymbol{V}(\boldsymbol{G})$ is called a dominating set of $\mathbf{G}$ if every vertex in $\boldsymbol{V}(\boldsymbol{G})-\boldsymbol{D}$ is adjacent to some vertex in $\boldsymbol{D}$. A set $\boldsymbol{D} \subseteq \boldsymbol{V}$ is called a distance $k$-dominating set of $G$ if each $x \in V-D$ is within distance $k$ from some vertex of $D$. In this paper, we determine the distance- $\boldsymbol{k}$ domination number for a given graph using the $k$-center and link vector concepts. Using the $k$-center concept we present some necessary and sufficient condition for distance- $k$ dominating set.
\end{abstract}

Keywords: Distance, radius, domination number, distance $k$-domination number, $k$-center, reachable set, link vector.

\section{INTRODUCTION}

Let $\boldsymbol{G}=(\boldsymbol{V}, \boldsymbol{E})$ be a simple graph. The distance between $\boldsymbol{u}$ and $\boldsymbol{v}$, denoted by $d(u, v)$, is the length of a shortest $\boldsymbol{u}-\boldsymbol{v}$ path. For a vertex $\boldsymbol{v} \in \boldsymbol{V}$ and a positive integer $k$, the $k$ neighborhood of $\boldsymbol{v}$ in $\boldsymbol{G}$ is defined as $\boldsymbol{N}_{\boldsymbol{k}}(\boldsymbol{v})=\{\boldsymbol{u} \in$ $\boldsymbol{V}(\boldsymbol{G}) / \boldsymbol{d}(\boldsymbol{u}, \boldsymbol{v})=\boldsymbol{k}\}$. For $\boldsymbol{k}=\mathbf{1}, \boldsymbol{N}_{\mathbf{1}}(\boldsymbol{v})$ is the neighborhood of $\boldsymbol{v}$ and simply denoted by $\boldsymbol{N}(\boldsymbol{v})$. Let $\boldsymbol{d}(\boldsymbol{x})=|\boldsymbol{N}(\boldsymbol{x})|$ be the degree of $G$ and $\boldsymbol{\delta}$ and $\Delta$ be the minimum and maximum degree of $\boldsymbol{G}$, respectively. The set $\boldsymbol{N}_{\boldsymbol{k}}[\boldsymbol{v}]=\boldsymbol{N}_{\boldsymbol{k}}(\boldsymbol{v}) \cup\{\boldsymbol{v}\}$ is called the closed $\boldsymbol{k}$ neighborhood $\boldsymbol{v}$ in $\boldsymbol{G}$.

For a connected graph $G$, the eccentricity $e(v)=$ $\max \{d(u, v): \forall u \in V(G)\}$ and the eccentric set $E(v)=\{u \in$ $V: d(u, v)=e(v)\}$. The minimum eccentricity among the vertices of $G$ is its radius and the maximum eccentricity is its diameter, which is denoted by $\operatorname{rad}(G)$ and $\operatorname{diam}(G)$, respectively. A vertex $v$ in $G$ is a central vertex if $e(v)=$ $\operatorname{rad}(G)$ and the subgraph induced by the central vertices of $G$ is the center $\operatorname{Cen}(G)$ of $G$. In this paper, we present the relation between distance- $k$ dominating set and $k$-center of the given graph. We study the binary operations $V, \wedge$ in [1]. Using these operations we construct algorithm to find the distance $k$ dominating set.

Definition 1.1: A set $D \subseteq V(G)$ is called a dominating set of $G$ if every vertex in $V-D$ is adjacent to some vertex in $D$.

The domination number $\gamma(G)$ is the minimum cardinality of a dominating set. We call the set of vertices as a $\gamma$-set if it is a dominating set with cardinality $\gamma(G)$.

Definition 1.2: A set $D \subseteq V(G)$ is called a distance $k$ dominating set of $G$ if $N_{k}[D]=V$. The distance $k$-domination number $\gamma_{k}(G)$ of $G$.

\section{2. k-center [1]}

Definition 2.1: Let $S$ be a subset of $V$ with $k$ vertices. Let $v \in$ $V$. Then the distance of $S$ from $v$ is defined as $d(S, v)=$ $\min \{d(x, v) / x \in S\}$. If $v \in S$ then $d(S, v)=0$. The eccentricity of $S$ is the maximum of $d(S, v)$ over all $v \in V$. That is, $e(S)=\max \{d(S, v) / v \in V\}$.Consider the family $F_{k}$ of the subset $S$ of $k$ vertices $(1 \leq k \leq n-1)$ of $G$. The $k$-center of the graph $G$ is the set $S^{*}$ of $k$ vertices of $G$ such that, $e\left(S^{*}\right)=$ $\operatorname{Min}\left\{e(S), S \in F_{k}\right\}$. This minimum eccentricity is called the radius of $k$-center and it is denoted by $r_{k}(G)$.

\section{Theorem 2.2}

Every central vertex with radius $k$ forms a distance $k$ dominating set.

Proof

Let $\mathrm{G}$ be a graph with radius $k$. Let $C(G)$ be the center of the graph $G$. Let $C(G)=\left\{v_{1}, v_{2}, \ldots \ldots, v_{m}\right\}$. If $v_{i} \in$ $C(G),(1 \leq i \leq m)$, then $\mathrm{e}\left(v_{i}\right)=\mathrm{k}$. Hence $d\left(v_{i}, v\right)=k$ for some $v$ in $V$. Therefore $d\left(v_{i}, v\right) \leq k$ for all $v \in V$ i.e, Each $v_{i}$ is with distance $k$ to all other vertices in $G$. Hence each $v_{i}$ can dominate all the vertices of $G$ with distance $k$. Hence every center vertex with radius $k$ forms a distance $k$-dominating set.

Theorem 2.3

For any connected graph with radius $k, \gamma_{k}(G)=1$.

Proof

Let $G$ be any connected graph. Let $k$ be the radius of $G$. Let $C(G)$ be the center of $G$. Then $e(v)=k$ for all $v \in$ $C(G)$. Let $v \in C(G)$ then $\max \{d(v, u)=k ; u \in V\}$. Also we have $d(v, u) \leq k ; u \in V$. Hence $v$ dominates every vertex within distance k. So $\gamma_{k}(G)=1$.

\section{Theorem 2.4}

For any connected graph $G, \gamma_{k}(G)=1$ if and only if there exists a vertex in $G$ with eccentricity $\leq k$.

\section{Proof}

Let $\mathrm{G}$ be any connected graph and $V(G)=\left\{v_{1}, v_{2}, \ldots \ldots, v_{n}\right\}$. Suppose that $\gamma_{k}(G)=1$. Let $D$ be a minimum distance $k$ dominating set. Let $x_{i} \in D$.

Case (i): If $r(G) \leq k \leq \operatorname{diam}(G)$, then there exists a vertex can dominate all the vertices within distance $k$. So that $d\left(v_{i}, v\right) \leq k \forall v \in V-\left\{v_{i}\right\}$. Which implies that $e\left(v_{i}\right)=$ $\max \left\{d\left(v_{i}, v\right), v \in V-\left\{v_{i}\right\}\right\}=k$.

Case(ii): If $k>\operatorname{diam}(G)$, then there exists a vertex $v_{i}$ can dominate all other vertices of $G$ with distance less than $k$. Hence $d\left(v_{i}, v\right)<k \forall v \in V-\left\{v_{i}\right\}$. Which implies that $e\left(v_{i}\right)=\max \left\{d\left(v_{i}, v\right), v \in V-\left\{v_{i}\right\}\right\}<k$. 
Conversely, assume that there exists a vertex with eccentricity $\leq k$. Let $v_{i}$ be a vertex with $e\left(v_{i}\right) \leq k$. Then obviously, $d\left(v_{i}, v\right) \leq k \quad \forall v \in V-\left\{v_{i}\right\}$. Hence $D=\left\{v_{i}\right\}$ can dominate all other vertices of $G$. Then $D$ is a minimum distance $k$-dominating set of $G$. Hence $\gamma_{k}(G)=1$.

\section{Theorem 2.5}

Every $k$-center of $\mathrm{G}$ with radius $i$ is a distance $i$ dominating set.

\section{Proof}

Let $G$ be any connected graph with $n$ vertices. Let $S_{k}$ be the $k$-center of $G$ with radius $i$. Hence $\left|S_{k}\right|=k$ and $e\left(S_{k}\right)=$ $i$. That is the distance of $S_{k}$ from the farthest vertex is $i$. Therefore, $S_{k}$ dominates the farthest vertex with distance $i$. Hence $S_{k}$ dominates all vertices of $\mathrm{V}$ within distance $i$ and so $S_{k}$ is a distance $i$-dominating set.

Definition 2.6: The set of all vertices of the graph $G$, from which the vertex $x$ is connected within a minimum distance $\lambda$ is called as a reachable set of $x$ within a distance $\lambda$ and is denoted as $R_{\lambda}(x)=\{y \in V / d(y, x) \leq \lambda\}$. Call this distance $\lambda$ as penetration.

Definition 2.7: Characterize each vertex as a $n$-tuple. Each place of $n$-tuple can be represented by a binary zero or one. Call this $n$-tuple as a link vector simply LV of a vertex.

Thus a link vector $\left(j_{1}, j_{2}, \ldots, j_{n}\right)$ represent a vertex $x_{j}$ where $j_{k}=1$ if $x_{k}$ is reachable within the penetration $\lambda$ from $x_{j}$ and zero otherwise. Denote a link vector of the vertex $x$ by $x^{\prime}$ and denote the set of all link vectors as $\Omega$.

If all the coordinate of a link vector of a vertex are equal to one then the link vector is said to be full and is denoted as (1). If all the coordinates of a link vector of a vertex are equal to zero then the link vector is said to be null and it is denoted by (0).

Definition 2.8: Let $\mathrm{G}$ be a graph. Let $\Omega$ be the set of LVs of all vertices. Define two binary operations $V$ (cup) and $\Lambda$ (cap) as follows:

$\vee, \wedge: \Omega \times \Omega \rightarrow \Omega$ by

$\left(a_{1}, a_{2}, \ldots, a_{n}\right) \vee\left(b_{1}, b_{2}, \ldots, b_{n}\right)=\left(c_{1}, c_{2}, \ldots, c_{n}\right)$

where $c_{i}=\max \left\{a_{i}, b_{i}\right\} \& i=1$ to $n$

$$
\left(a_{1}, a_{2}, \ldots, a_{n}\right) \wedge\left(b_{1}, b_{2}, \ldots ., b_{n}\right)=\left(c_{1}, c_{2}, \ldots ., c_{n}\right)
$$

where $c_{i}=\min \left\{a_{i}, b_{i}\right\} \& i=1$ to $n$

\section{Theorem 2.9}

Let $G$ be a graph with $n$ vertices and $r_{k}(G)=i$. Let $D \subseteq V$ of $k$ vertices $(1 \leq k \leq n-1)$. Then $D$ is a distance $i$ dominating set if and only if $D$ is a $k$-center.

\section{Proof}

Let $D \subseteq V$ be a set of $k$ vertices with $r_{k}(G)=i$. Suppose that $D$ is a distance $i$-dominating set. Then there exists a vertex $v$ in $D$ such that $d(u, v) \leq i$, for every $u \in V-D . \therefore e(D)=$
$i=r(D)$. It implies that $D$ is a $k$-center. Conversely, suppose that $D$ is a $k$-center with radius $i$. By theorem $2.5, D$ is a distance $i$ - dominating set.

Now we take $i=1$, then we have the following corollary.

\section{Corollary 2.10 [1]}

In any graph $G$ with radius 1 , a set $D$ of $k$ vertices $1 \leq$ $k \leq n-1$ is a dominating set if and only if $D$ is a $k$-center.

\section{Theorem 2.11}

Let $G$ be a graph with $n$ vertices. Then $\mathrm{V}_{j=1}^{k} x_{j}{ }^{\prime}$ is full for a least integer $k$ in $G$ for $\lambda=i$ if and only if $D=$ $\left\{x_{1}, x_{2}, \ldots, x_{k}\right\}$ is a minimum distance $i$-dominating set.

\section{Proof}

Consider the amount of penetration $\lambda=i$. Suppose that $\mathrm{V}_{j=1}^{k} x_{j}{ }^{\prime}$ is full where $x_{j}{ }^{\prime}$ is the LV of $x_{j}$. Take $x_{j}{ }^{\prime}=$ $\left(x_{j_{1}}, x_{j_{2}}, \ldots, x_{j_{n}}\right)$ for a least integer $k$. NowV $V_{j=1}^{k} x_{j}{ }^{\prime}=$ $\left(x_{1_{1}}, x_{1_{2}}, \ldots, x_{1_{n}}\right) \vee\left(x_{2_{1}}, x_{2_{2}}, \ldots, x_{2_{n}}\right) \vee \ldots \vee$ $\left(x_{k_{1}}, x_{k_{2}}, \ldots, x_{k_{n}}\right)$. Since $\mathrm{V}_{j=1}^{k} x_{j}{ }^{\prime}$ is full, then $\left(x_{1_{1}}, x_{1_{2}}, \ldots, x_{1_{n}}\right) \vee\left(x_{2_{1}}, x_{2_{2}}, \ldots, x_{2_{n}}\right) \vee \ldots \ldots \vee$ $\left(x_{k_{1}}, x_{k_{2}}, \ldots, x_{k_{n}}\right)=(1,1, \ldots .1)$. Hence $D=\left\{x_{1}, x_{2}, \ldots, x_{k}\right\}$ dominates $V$ and it is a minimum distance $i$-dominating set. Since $k$ is minimum.

Conversely, suppose that $D=\left\{x_{1}, x_{2}, \ldots, x_{k}\right\}$ is a minimum distance $i$-dominating set. Then a vertex not in $D$ is adjacent to at least one vertex of $D$ within distance $\lambda=i$. That is, $d(D, y) \leq i \forall y \in V$ - $D$. Thus all coordinates of any one of $x_{1}{ }^{\prime}, x_{2}{ }^{\prime}, \ldots . ., x_{k}{ }^{\prime}$ is 1 . Hence $x_{1}{ }^{\prime} \vee x_{2}{ }^{\prime} \vee \ldots . . \vee x_{k}{ }^{\prime}$ is full, that is $\bigvee_{j=1}^{k} x_{j}{ }^{\prime}$ is full. It completes the proof.

\section{Theorem 2.12}

Let $G$ be a graph with $n$ vertices. Then there exists a vertex whose link vector is full with $\lambda=k$ if and only if $\gamma_{k}(G)=1$.

\section{Proof}

Let $G$ be a graph with $n$ vertices. Suppose that there exists a vertex $v_{i}$ whose link vector is full with penetration $k$. That is, the $j^{\text {th }}$ coordinate of $v_{i}{ }^{\prime}$ is 1 for every $j(1 \leq j \leq n)$. Hence the vertex $v_{i}$ is reachable to all other vertices of $G$ with penetration $k$. Hence this vertex $v_{i}$ alone forms a distance $k$ dominating set. Hence $D$ is a minimum distance $k$-dominating set and so $\gamma_{k}(G)=1$. Conversely, assume that $\gamma_{k}(G)=1$. Let $D$ be a $\gamma_{k}$-set of $G$. Take $D=\left\{v_{i}\right\}$. Then the vertex $v_{i}$ dominates all other vertices within distance $k$. Hence $v_{i}$ is reachable to all other vertices of $G$ with $\lambda=k$. Then the LV $v_{i}^{\prime}$ of $v_{i}$ is full.

\section{Theorem 2.13}

If $r(G) \leq k \leq \operatorname{diam}(G)$, then there exist a LV $x_{i}{ }^{\prime}$ which is full with $\lambda=k$. 


\section{Proof}

Let $G$ be a graph with $n$ vertices. Assume that $r(G) \leq k \leq \operatorname{diam}(G)$. Then there exists a vertex $x_{i}$ of $G$ with eccentricity $k$. By theorem $2.4, \gamma_{k}(G)=1$. Then by theorem $2.12, x_{i}{ }^{\prime}$ is full with $\lambda=k$.

\section{Theorem 2.14}

Let $G$ be a connected graph with $n$ vertices. Then $\wedge_{i=1}^{n} x_{i}{ }^{\prime}$ is full with $\lambda=1$ if and only if $G$ is complete.

\section{Proof}

Let $G$ be a connected graph with $n$ vertices. Suppose that $\bigwedge_{i=1}^{n} x_{i}{ }^{\prime}$ is full with $\lambda=1$.

$$
\text { Then } \quad \wedge_{i=1}^{n} x_{i}{ }^{\prime}=\min \left[\left(x_{1_{1}}, x_{1_{2}}, \ldots, x_{1_{n}}\right) \wedge\right.
$$$$
\left(x_{2_{1}}, x_{2_{2}}, \ldots, x_{2_{n}}\right) \wedge \ldots . \wedge\left(x_{n_{1}}, x_{n_{2}}, \ldots, x_{n_{n}}\right)=(1,1, \ldots, 1) \text {. }
$$
Since the $j^{\text {th }}$ coordinate of $x_{i}$ is full for all $i, j(1 \leq i, j \leq n)$, then the vertex $x_{i}$ is reachable to all other vertices. Hence $G$ is complete.

$$
\text { Conversely, Take } \bigwedge_{i=1}^{n} x_{i}{ }^{\prime}=\min \left[\left(x_{1_{1}}, x_{1_{2}}, \ldots, x_{1_{n}}\right) \wedge\right.
$$
$\left.\left(x_{2_{1}}, x_{2_{2}}, \ldots, x_{2_{n}}\right) \wedge \ldots \wedge\left(x_{n_{1}}, x_{n_{2}}, \ldots, x_{n_{n}}\right)\right]$. Since $G$ is complete, link vector of every vertex is full. Hence $\Lambda_{i=1}^{n} x_{i}{ }^{\prime}$ is full. Thus the link vector concept is very useful to prove many results.

\section{Algorithm 2.15}

Algorithm to find a minimum distance $i$-dominating set

Input. A graph $G=(V, E)$ with $V(G)=\left\{x_{1}, x_{2}, \ldots, x_{n}\right\}$ with distance matrix and $\operatorname{diam}(G)=d$. Find all reachable sets $R_{\lambda}\left(x_{j}\right)$ of $x_{j}$ and find the link vector $x_{j}^{\prime}$ of $x_{j}$

Output. Minimum distance $i$-dominating set.

Step 1. $i=1$ to $d$
Step 2.

2.1. Take $w^{\prime}$ is the LV of $w$. Initialize $w^{\prime} \leftarrow(0)$ and $D=\emptyset$

$$
\begin{array}{r}
\text { 2.2. For } j=1 \text { to } n \\
w^{\prime}=w^{\prime} \vee x_{j}^{\prime} \\
D=D \cup\left\{x_{j}\right\}
\end{array}
$$

2.3. If $w^{\prime}$ is not full then go to step 3.2

2.4. Print $D$ is a minimum distance $i$-dominating set.

Otherwise go to step 3.2

\subsection{Go to step 1.}

This algorithm finds $d$ number of minimum distance $i$ dominating sets, for $i=1$ to $d$. It works with a for loop $j=1$ to $n$, for a fixed $i$. To find a minimum distance $i$-dominating set this algorithm works with the operations $V$ and $U$ in $2 n$ times. Totally it works in $2 n d$ times and so it is a polynomial time algorithm.

\section{REFERENCES}

[1] A. Anto Kinsley and S. Somasundaram, Domination based algorithm to k-center problem, Journal of Discrete Mathematical Sciences and Cryptography, Vol. 9 (2006), No.3, pp. 403-416.

[2] F. Buckley and F. Harary, Distance in Graphs, Addison - Wesley Publishing Company, New York, (1990).

[3] G. Chartrand and P. Zhang, Introduction to Graph Theory, Tata McGrew Hill Education Private Ltd, New Delhi (2006), 327-333.

[4] T. W. Haynes, S. T. Hedetniemi and P.J. Slater, Fundamentals of Domination in Graphs, Marcel Dekker, Inc., New York, (1998).

[5] P. J. Slater, Maximin facility location, J. Res. Net Burstandards, 79B, (1975), 107-115.

[6] H. S. Wilf, Algorithm and Complexity, Prentice - Hall International, Inc., U.S.A. (1986). 